\title{
ASSESSING DRIVER FATIGUE AS A FACTOR IN ROAD ACCIDENTS
}

\author{
William Sirois, Todd Dawson, Martin Moore-Ede, \\ Acacia Aguirre, Udo Trutschel, \\ Circadian Technologies, \\ Stoneham, MA USA \\ E-mail: bsirois@circadian.com
}

\begin{abstract}
Summary: Fatigue is one of the most pervasive yet under-investigated causes of human-error-related driving accidents, incidents, and injuries. Several studies suggest that 25-30\% of driving accidents are fatigue related (Horne et al., 1995). However, government reports estimate that only $1-4 \%$ of crashes may be attributable to the driver falling asleep or being drowsy, based largely on data derived from police reports recorded at these accidents (Cummings et al., 2001). The reason for this wide disparity is that there is no simple tool or objective way for investigators to collect the (right) data needed to correlate accidents with fatigue. To bridge this gap, a diagnostic survey instrument was developed, along with a weighted risk model based on Fuzzy Scalable Monotonic Chaining (FSMC), to help investigators readily determine (by standardized criteria and with high probability) the role of fatigue as a causal factor in driving accidents.
\end{abstract}

\section{INTRODUCTION}

The investigators of road accidents are confronted with a wide spectrum of possible causes, but generally have a limited amount of data to work with (Cummings et al., 2001; Horne et al., 1995). They must thus subjectively decide which factors are directly or indirectly correlated to the cause of the accident. While technical failure, excessive speed, or alcohol-related impairment are well accepted as causes of accidents, little attention is given to assessing the risk of fatigue as a contributing factor unless there is obvious evidence, such as a gradual drifting off the road with no other apparent causes. An approach for revealing any fatigue-related contribution to an accident would provide a more realistic picture of the situations under investigation, and should broaden the acceptance of fatigue-mitigating measures in the transportation industries.

In general, fatigue can be characterized by many features, such as a decrease in mental and physical performance, reduced vigilance, loss of cognitive and logical reasoning skills, impaired judgment, reduced motor coordination, slower reaction time, inability to process information, loss of concentration and perception, loss of short-term memory, absentmindedness, mental lapses, uncharacteristic mistakes, erratic behavior, and procedural deviations, etc. The resulting consequences of fatigue for businesses can thus include increased turnover and absenteeism, reduced driver morale, poorer labor relations, increased healthcare costs, reduced productivity and customer service quality, reduced operating efficiency, higher accident and incident rates, and increased overall costs, risks, and liabilities. Thus, fatigue-related accidents and incidents cost businesses large sums of money, both in terms of personal injuries and fatalities, as well as in property damage and litigation. Moreover, the societal costs of fatigue-related accidents are compounded many times over. 
To have the ability, then, to truly expose the contribution of fatigue to the overall portfolio of preventable safety accidents and incidents is of critical importance to justify the resources required for addressing the issue - and recovering the costs in lives and dollars that are currently being accepted as part of life or of doing business. To achieve this goal, it is obviously necessary to collect the type of data that will objectively correlate with the features, or factors, of fatigue. These include direct or indirect influence by circadian time of day, irregular work and sleep hours, extended hours of work, excessive overtime, disrupted or inadequate sleep, by the time since the last consolidated sleep episode, by cumulative sleep deprivation, by boring and monotonous tasks and work environments, by health issues, psychosocial factors, and/or stress. These factors were captured in a new survey instrument, and were analyzed by both an established predictive software simulation model, and by a recently developed fuzzy fatigue risk estimation tool.

\section{METHOD}

To determine the probability of fatigue as the causal factor of an accident requires the capturing of hard numerical data as well as of verbal indicators. Thus, a fuzzy reasoning approach may be the best algorithm for probability calculations involving numerical and verbal attributes, particularly with the relatively uncertain circumstances of an accident event. Fuzzy reasoning, in general, requires a set of rules which increase dramatically in a complex problem, such as the determination of fatigue probability in an accident. To accumulate as much evidence as possible for identifying the fatigue contribution in an accident, an additive form of fuzzy reasoning was therefore employed. This is called Fuzzy Scalable Monotonic Chaining (FSMC) and was first introduced by Cox (1994).

The FSMC approach can handle any number of rules and still maintain the important relationship between the underlying rules and the overall risk factors for fatigue. Figure 1 shows the principle architecture of the FSMC model that was used to evaluate the fatigue-related risk factors to transportation accidents, based on a simple questionnaire. The response to each of the survey questions was translated into a rule for the FSMC model. The fatigue-related risk factors formulated into rules were then mapped by this 'Fuzzy set' into an intermediate 'Scaled Risk Level.' The result of this mapping is a numeric value (e.g., 451 and 398) indicating the degree of fatigue risk obtained by the answer to a specific survey question. The monotonic reasoning result for each rule is then added to calculate the 'Total Risk.' This numeric value of the 'Total Risk' is then used to obtain the contribution of the 'Fatigue Risk,' or probability, of an accident by a fuzzy membership function. The FSMC method allows display of individual risks for each question of the survey, as well as any follow-up calculations.

For example, Figure 2 shows the fuzzy sets associated with the first question of the survey (the time of the accident) and a fatigue score calculation by the validated Circadian Alertness Simulator (Moore-Ede et al., 2004; Heitmann et al., 2005). This is an established predictive software model, known as CAS, for determining fatigue risk. 


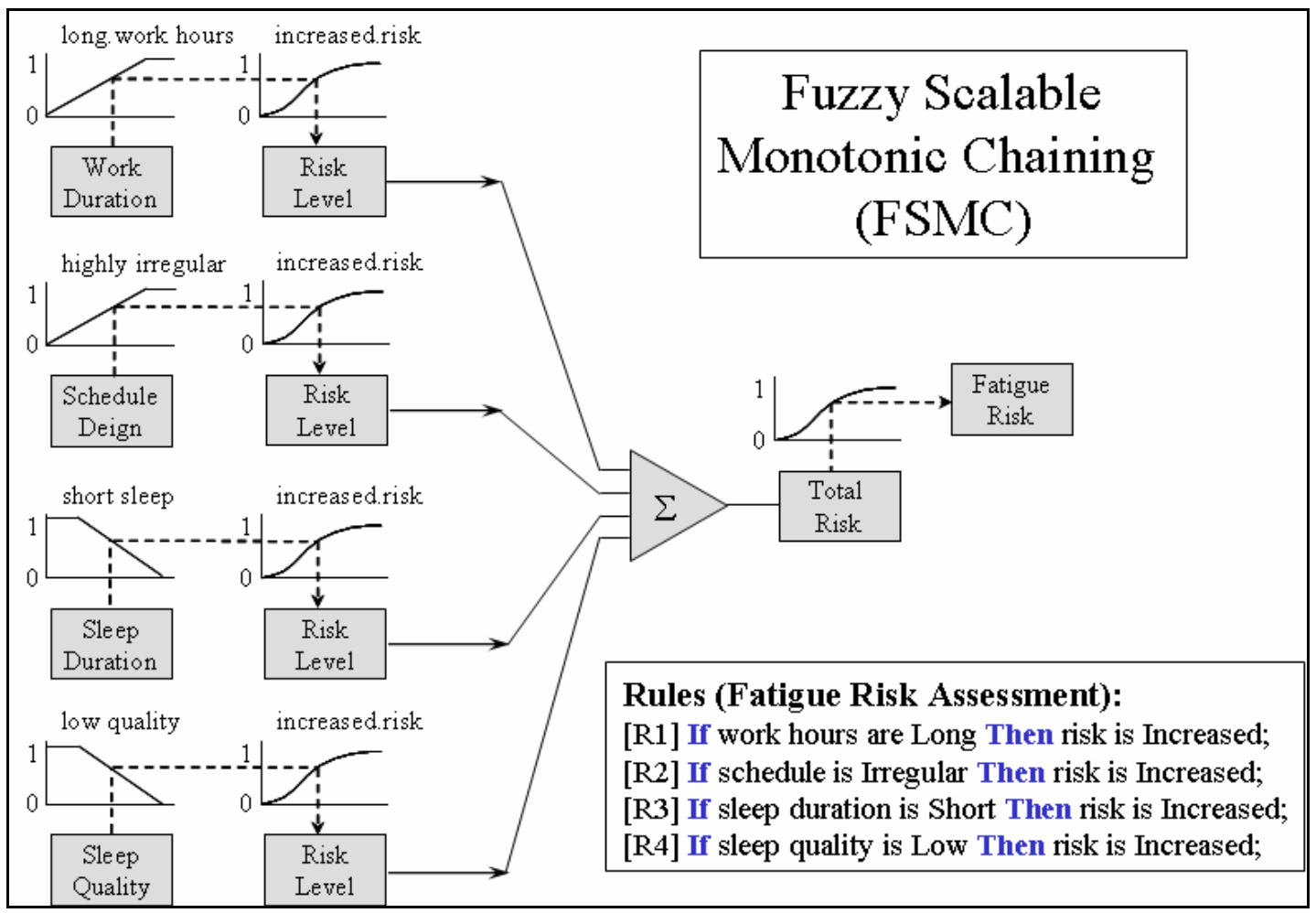

Figure 1. General Concept of Fuzzy Scalable Monotonic Chaining (FSMC)

\section{Rule 1: If "Accident Time" is known then "Accident Risk" can be estimated;}
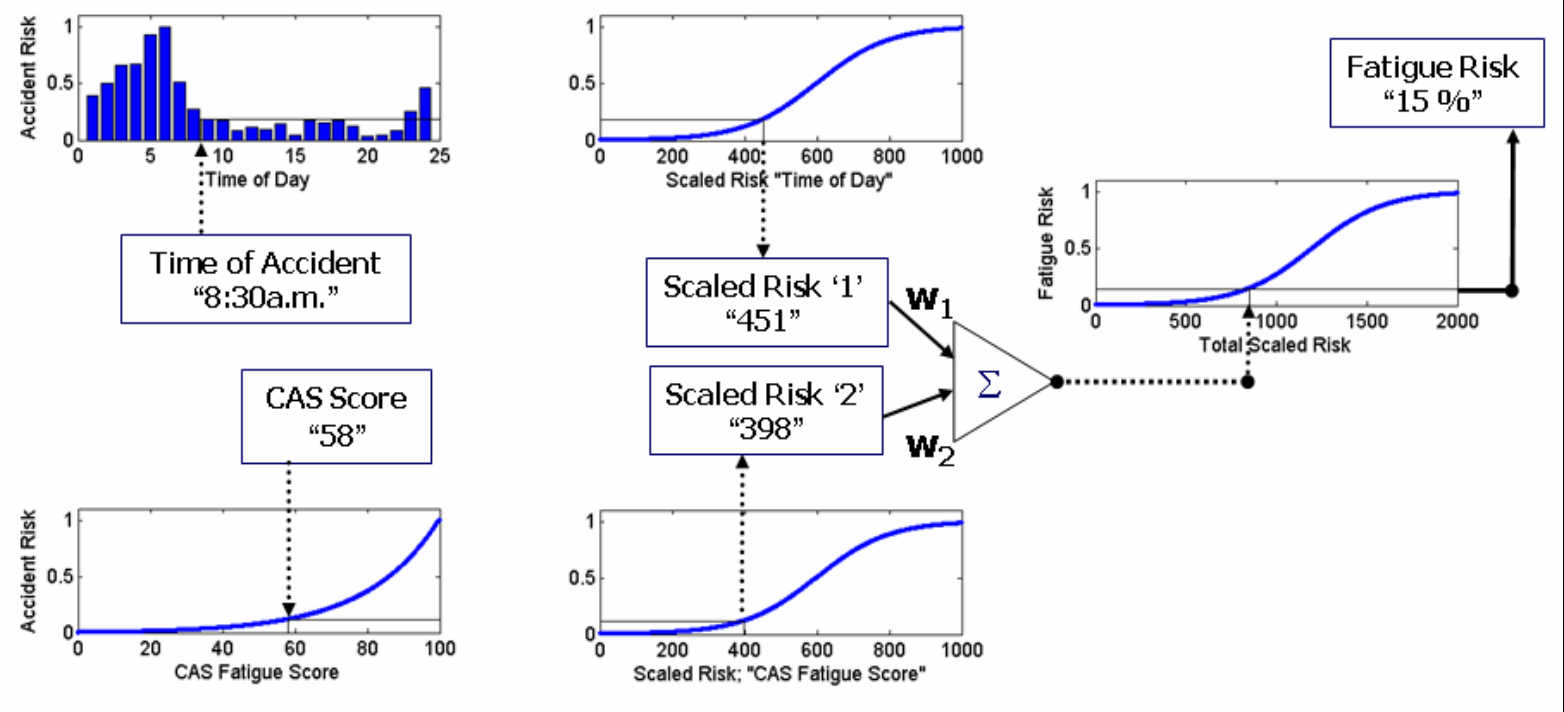

Rule 2: If "CAS Fatigue Score" is High then "Accident Risk" is increased;

Figure 2. Rule based fuzzy mapping of normalized risk, combined into a total risk estimate, and mapped to an overall fatigue probability (for a two rule case). 
The graph in the upper left corner of Figure 2 displays the well-documented occurrence of road accidents as function of time of day. This accident probability can be directly scaled to a normalized risk level that can be compared with other data. The graph in the lower left corner shows the accident probability as a function of the CAS fatigue score (Heitmann et al., 2005). This CAS fatigue score summarizes and evaluates the sleep/wake history of the days before the accident and converts this into an accident-risk estimation. Both risk factors (rules) are mapped to a scale of 0 to 1000, which is arbitrary but works well for a large number of rules. This scaling indicates if all ' $N$ ' rules are at maximum results in a Total Risk score of ' $N$ ' $x 1000$. In this example, (Figure 2), we consider the fuzzy mapping of two rules (' $\mathrm{N}$ '=2). Therefore, the graph on the right side in Figure 2 is scaled from 0 to 2000. It is important to have an accident investigation concept with an adaptable number of rules. Sometimes, certain information can't be recovered after an accident, and therefore, the number of rules has to be reduced. In other cases, additional information is available and the number of rules has to be increased. It should be obvious that the shape of the fuzzy sets allows adjustments for how each contributing risk of each rule is interpreted. With the FSMC approach, an arbitrary combination of verbal or numerical inputs, such as time of accident, day of shift, weather conditions, and calculated CAS fatigue scores can be analyzed by fuzzy rules. Each fuzzy module with only one input generates an intermediate risk (or probability) value. The resulting fatigue probability can thus be a combination of the intermediate values based on their degrees of influence. From a practical perspective, some input parameters will be less influential than others in the generation of fatigue probability scores. In a generalized concept, the relative importance among inputs can be scaled by means of pre-defined weights (denoted as ' $\mathbf{w}_{\mathbf{1}}$ ' and ' $\mathbf{w}_{\mathbf{2}}$ ', in Figure 2). Parameters of less importance were assigned smaller weights and parameters of greater importance were assigned higher weights. The cumulative fatigue-related risk is then a simple weighted superimposition of individual risk components. This prototype method was then tested for veracity against five case study accidents, and compared to the results derived using the CAS model as the test standard.

\section{ACCIDENT CASES USED FOR TESTING THE FSMC PROTOTYPE}

Note: The complete work and sleep history of the individuals involved in the following case study examples was available for at least the first 3 days prior to the accident.

Case 1. A 45-year old male truck driver (TD1) who had been working at this same company for more than 15 years, had arrived earlier than usual (approximately 6:00 AM) for a meeting. Following the meeting, his first assignment was to move his truck to the loading terminal. At 8:15 AM, TD1 collided with another truck 'standing' in the yard. At the time of the accident, TD1 was working his $5^{\text {th }}$ day shift. His sleep and work pattern can be characterized as regular. His sleep during the night before the accident was shortened by two hours because of the early meeting. TD1 also had a history of some health and sleep problems.

Case 2. This collision occurred at approximately 11:35 AM. According to the police accident report, Motor Vehicle (MV2) Driver was stopped at a red light. Truck Driver (TD2) approached the intersection but failed to stop, rear-ending the MV2 vehicle. There were no pre-accident skid marks, which indicated that TD2 did not attempt any hard braking to avoid the collision. At the time of the accident, TD2 was working his second day following two days off. However, his 
sleep and work pattern can be characterized as highly irregular. TD2's last sleep before the accident was during the previous day and started at 8 PM. The sleep duration was only 4 hours.

Case 3. The collision occurred at approximately 8:30 PM. Motor Vehicle (MV3) was traveling westbound. Truck Driver (TD3) lost control of his vehicle and swerved into the westbound lane of traffic, where it collided with the vehicle of MV3. At the time of the accident, TD3 was returning home after a trip covering 3,200 miles over several days of driving. He had not slept for more than 27 hours prior to the accident, and had a history of frequent sleep deprivation caused by his schedule. His sleep and work pattern can be characterized as highly irregular.

Case 4. Truck driver (TD4) was traveling north on an Interstate. While on a 7-percent downgrade, his tractor-trailer ran off the right side of the road as the roadway curved to the left. At the time of the accident (9:10 PM) TD4 was working a 4th consecutive day after two days off. His sleep pattern can be characterized as close to regular. His work hours were during the daytime, but the shifts were split.

Case 5. This accident involved a collision between a tractor-trailer operated by driver (TD5b) and an automobile driven by AD5a at approximately 12:28 PM. TD5b's tractor-trailer was traveling northbound and making a right turn into a rest area when struck by AD5a's car as AD5a attempted to pass on the right. Driver AD5a stated that driver TD5b's left turn signal was on as he attempted to pass the truck, giving him the impression that the truck was turning left. However, truck driver TD5b stated that at no point was his left turn signal ever on, only his right turn signal as he prepared to turn right into the rest area. Because the circumstances of the accident were in dispute, the fatigue levels of both drivers were analyzed. The investigation of driver AD5a and driver TD5b are labeled as Case 5a and Case 5b, respectively.

Case $5 a$. The accident occurred after automobile driver AD5a had completed his third consecutive night shift of the week, at approximately 5 hours prior to the time of the accident. During the days prior to the accident, his sleep pattern was split, and completely the reverse of his pattern during his days off. AD5a had slept approximately 24 hours before the time of the accident, and his sleep prior to that was 48 hours prior to the accident.

Case $5 b$. At the time of the accident TD5b was on duty and driving his truck. He worked a regular day shift on weekdays, beginning most trips between 4 AM and 6 AM in the morning, and finishing work before 6:30 PM. During the week of the accident he had taken two days off, and was working his third consecutive day shift (that week) at the time of the accident. His sleep and work pattern can be characterized as regular. Noticeable was the difference in sleep length between his working days and his days off.

\section{RESULTS}

These known accident examples were analyzed by two methods: (1) the Circadian Alertness Simulator (CAS) to establish the standard, and (2) the FSMC method described in this paper. The results are depicted in Figure 3. In the bottom half of Figure 3, the CAS results are presented on a scale of 0 to 100 , with 100 being extreme fatigue. Based on the CAS outcome, which is a validated, predictive software model, the fatigue contribution to the accident (for Case 1 and 
Case $5 \mathrm{~b}$ ) was determined to be unlikely. For Case 4, the probability of fatigue was low, and for Cases 2, 3, and 5a the probability was very high. In the upper half of Figure 3, the FMSC results are presented on a scale of 0 to 'N'x1000. The fatigue contribution of Case $5 \mathrm{~b}$, as predicted by the FMSC method, was also determined to be unlikely. Case 4 also correlated with the CAS results as low probability. Case 5a and Case 1 showed moderate probability, and Cases 2, and 3 suggested high probability. Thus, the results from both approaches appear to have correlated fairly well. Only the fatigue rating of Case 1 was significantly different. This is not surprising considering the fact that there were health and sleep problems involved, which are difficult to capture and difficult to simulate in the CAS model, but are detectable with the FMSC approach. Overall, both methods demonstrated an encouragingly good agreement, considering the different basic nature (hard numerical values for CAS versus fuzzy combinations of numeric and verbal attributes for FMSC) of these two approaches.

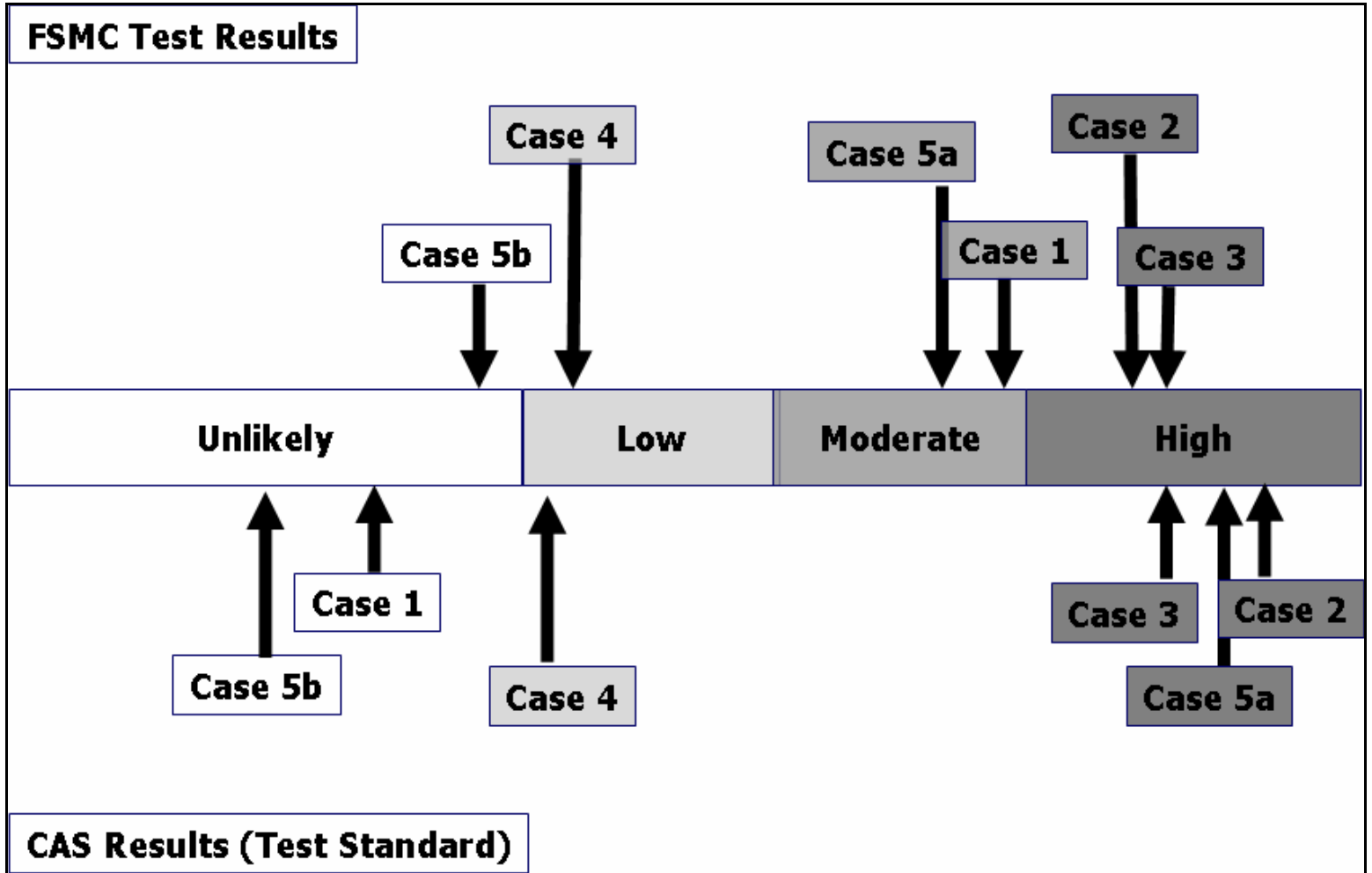

Figure 3. Probability of fatigue contribution (Unlikely, Low, Moderate, and High) to an accident using two approaches: (1) Standardized CAS results (lower half) on a scale from 0 to 100 ; (2) FSMC test results (upper half) on a scale from 0 to ' $N$ ' $\times 1000$.

\section{CONCLUSIONS}

These test results suggest that the fuzzy mapping method shows great promise in providing a simple tool that investigators can use to realistically determine fatigue as a causal factor in driving accidents. To further validate this new model, a comparison between 10 additional accident cases (five fatigue and five non-fatigue related) from a National Transportation Safety Board safety study (NTSB, 1995) will be presented on a poster board exhibit at the Driver Assessment 2007 conference. The conclusions obtained from the on-site NTSB accident 
investigators will hopefully serve to further refine and fine-tune this fuzzy mapping approach. The ultimate objective is to achieve a reliable, easy to use, and accepted tool for the determination of the fatigue contribution to an accident. To support the development of such an accident investigation tool based on Fuzzy Scalable Monotonic Chaining (FSMC), several operating companies are currently collecting survey data to help validate this approach for determining fatigue as a causal factor.

\section{REFERENCES}

Cummings, P., et al. (2001). Drowsiness, countermeasure to drowsiness, and the risk of a motor vehicle crash, Injury Prevention, 7, 194-199.

Horne, J., Reyner, L. (1995). Sleep related vehicle accidents, British Medical Journal, 310, 565567.

Cox, E. (1994). The Fuzzy Systems Handbook. Academic Press, Inc.

Moore-Ede, M., Heitmann, A., Guttkuhn, R., Trutschel, U., Aguirre, A., Croke, D. (2004). Circadian Alertness Simulator for Fatigue Risk Assessment in Transportation: Application to Reduce Frequency and Severity of Truck Accidents. Aviation, Space, and Environmental Medicine, 75(1), A107-A118.

Heitmann, A., Guttkuhn, R., Croke, D., Moore-Ede, M. (2005). Innovative fatigue management approach in the trucking industry. Proceedings of the Third International driving Symposium on Human Factors in Driver Assessment, Training and Vehicle Design, Rockport, Maine.

NTSB (National Transportation Safety Board), Safety Study (1995). Factors that affect fatigue in heavy truck accidents. Safety Study. Volume 2: Case Summaries. 